\title{
Electronic structure and magnetism of strained bec phases across the fcc to bec transition in ultrathin Fe films
}

\author{
Alberto Calloni, ${ }^{1, *}$ Giulia Berti, ${ }^{1}$ Gianlorenzo Bussetti, ${ }^{1}$ Guido Fratesi, ${ }^{2}$ Marco Finazzi, ${ }^{1}$ Franco Ciccacci, ${ }^{1}$ and Lamberto Duò ${ }^{1}$ \\ ${ }^{1}$ Dipartimento di Fisica, Politecnico di Milano, Piazza Leonardo Da Vinci, 32, 20133 Milano, Italy \\ ${ }^{2}$ ETSF, CNISM, Dipartimento di Fisica, Università degli Studi di Milano, via Celoria 16, I-20133 Milano, Italy
}

(Received 24 September 2016; published 30 November 2016)

\begin{abstract}
We investigated the electronic structure of the bcc metastable phases involved in the fcc to bcc transition of Fe. Ultrathin Fe films were grown on a 2-monolayer (ML) Ni/W(110) substrate, where a fcc lattice is stabilized at low Fe coverages and the transition proceeds through the formation of bcc nuclei showing a specific "Kurdjumov-Sachs" orientation with the substrate. A comprehensive description of the electronic structure evolution is achieved by combining spin-resolved UV photoemission spectroscopy and $a b$ initio calculations. According to our results, an exchange-split band structure is observed starting from $2 \mathrm{ML}$ of $\mathrm{Fe}$, concomitant with the formation of ferromagnetic bcc nuclei. Continuous modifications are observed in the spin-resolved photoemission spectra for increasing Fe coverage, especially for what concerns the minority states, possibly indicative of the progressive relaxation of the strained bcc phase starting from the bcc/fcc interface.
\end{abstract}

DOI: 10.1103/PhysRevB.94.195155

\section{INTRODUCTION}

The present work focuses on the electronic and magnetic properties of ultrathin, epitaxial Fe films. In standard conditions of temperature and pressure $(273 \mathrm{~K}$ and $100 \mathrm{kPa})$, bulk Fe shows a bcc, ferromagnetic crystal structure, called the $\alpha$ phase [1]. Unstable Fe phases, such as fcc $\gamma$-Fe (austenite), can be realized in very thin films grown on proper materials, typically with a fcc crystal structure, where Fe atoms are forced to adopt the same atomic arrangement of the substrate (pseudomorphic growth). As a consequence of the loading of elastic energy in the films, at larger thicknesses $\mathrm{Fe}$ invariably relaxes towards its bulk configuration. According to the literature [2,3], this is likely to occur through a diffusionless phase transformation, called a martensitic transformation. In principle, the fully relaxed bcc bulk structure can be obtained from the fcc one by applying a continuous tetragonal deformation of the unit cell (the so-called Bain strain). In practice, more complicated atomic displacements are observed following, e.g., the so-called Nishiyama-Wassermann (NW) or Kurdjumov-Sachs (KS) pathways [2]. Being a first-order phase transformation, the martensitic transformation implies the formation and evolution of bcc nuclei within a fcc host. The bcc nuclei are usually strained and then undergo a gradual relaxation towards the bulk equilibrium structure [4]. In some particular systems, the relaxation is more abrupt and the strained nuclei can be even considered as an intermediate or precursor phase within the martensitic transformation [5].

Historically, the research on thin Fe films focused on the study of the Fe fcc phase, which might be characterized, owing to its low dimensionality and crystal structure, by a ferromagnetic behavior with enhanced magnetic moments [6-8].The stabilization and the determination of the magnetic behavior of such phase, however, proved to be a formidable task. The use of different substrates (with different lattice parameters and surface crystallographic orientation) and different growth methodologies (molecular beam epitaxy either

\footnotetext{
*alberto.calloni@polimi.it
}

at cryogenic substrate temperature or at room temperature vs pulsed laser deposition or electrochemical deposition), for instance, gave rise to a complex phenomenology [9-11]. One-layer-thick Fe films [or Fe monolayers (ML)] on notmagnetic fcc (hcp) substrates such as $\mathrm{Cu}$ [12] or $\mathrm{Ir}(\mathrm{Ru})$ [13], are characterized either by an antiferromagnetic ordering, or a rather complex noncollinear arrangement of the local magnetic moments [14], while a paramagnetic behavior has been reported for $\mathrm{Au}$ [15]. Thicker Fe films have been also investigated, the most known example being $\mathrm{Fe}$ on $\mathrm{Cu}(001)$ for Fe thicknesses in the 5-10 ML range, which shows a peculiar behavior with a ferromagnetic top bilayer sitting on an antiferromagnetic bulk [16,17]. One major complication to the complete understanding of the magnetic behavior of thin films is, however, the magnetic response of the bcc phase. Past investigations with averaging techniques such as photoemission spectroscopy (PES) or magneto-optical Kerr effect (MOKE), for instance, detected a not-nil magnetic signal on thin Fe films and related it to the appearance of either low- or high-spin ferromagnetic $\gamma$ (i.e., fcc) phases [18,19]. However, accurate structural studies performed by scanning tunneling microscopy revealed, also in those cases, the formation of bcc nuclei, calling for a more complex interpretation of the previous experimental results (see, for instance, the ongoing discussion on the growth of $\mathrm{Fe}$ on $\mathrm{Cu}(001)$ [20,21] or $\mathrm{Cu}(111)$ [3]). Our goal is, therefore, to improve, by means of electron spectroscopies, and also with spin resolution, the present understanding of the electronic and magnetic structure of thin Fe films, starting from a more accurate investigation of the spectroscopic signal which is expected from the fcc phase and from the bcc nuclei, and from an assessment of the overall sensitivity of averaging techniques such as PES to the onset of the phase transformation. From a slightly different perspective, we foresee a contribution from such investigation also to the understanding of the electronic structure of Fe films where a distorted lattice is "stabilized" by means of alloying. Ordered alloys of, e.g., FePt [22] or FeCo [23] are notable examples. In those cases, characterized by the tetragonal distortion of the bcc Fe unit cell, a ferromagnetic behavior is obtained, together with a high uniaxial magnetocrystalline anisotropy, usually in 
the direction perpendicular to the film plane. Such systems are currently being researched for applications in magnetic and magneto-optical recording [24] and in spintronic devices such as magnetic random access memories (MRAMs) [25]. Recently, the study reported in Ref. [26] has shown the feasibility of an irradiation-induced fcc to bcc transformation in thick, nonmagnetic fcc Fe films alloyed with $\mathrm{Ni}$ that would allow, in perspective, for the magnetic patterning of a metastable Fe substrate by focused ion or electron beams.

In the present study, a well-defined crystallographic structure is obtained for the Fe films by following the strategy of Ref. [2]. Instead of the well-investigated (but still problematic) $\mathrm{Cu}$, we chose a Ni substrate for our electronic and magnetic characterization. Although the $\mathrm{Cu}$ lattice parameter is closer to that characteristic of $\gamma$-Fe $(3.52 \AA)$ [1], the lattice mismatch in the Ni case is tolerable (below 3\%) and, more important, the growth of Fe produces a much more controlled morphology (i.e., a nearly layer-by-layer growth at low coverages), thanks to a negligible $\mathrm{Fe} / \mathrm{Ni}$ interdiffusion, even at room temperature (RT) [2,27], and similar surface energies [28]. Magnetic bias from $\mathrm{Ni}$ is reduced by using ultrathin $\mathrm{Ni}$ films. In particular, we used a 2-ML Ni/W(110) multilayer structure. As it will become apparent in the following, the choice of a bcc substrate (W in the present case) is not accidental: while it does not prevent the formation of a fcc Ni surface [29], it steers the fcc to bcc transformation of Fe by selecting fewer bcc variants, therefore simplifying the interpretation of the experimental results.

The paper is organized as follows. After a description of the methods in Sec. II, we present our results in Sec. III: in the first part, the morphological evolution of the Fe surface is described by means of low-energy electron diffraction (LEED). The electronic structure is then investigated by means of spinresolved photoemission spectroscopy (SRPES). Eventually, in Sec. IV the results are discussed in light of (i) the available information on bulk Fe and (ii) ab initio calculations devised to link the observed evolution to the structural modifications occurring within the bcc phase.

\section{METHODS}

\section{A. Growth and spectroscopic characterization}

Our measurements were performed in an ultrahigh-vacuum (UHV) system with a base pressure in the low $10^{-8} \mathrm{~Pa}$ range [30]. The $\mathrm{W}(110)$ single crystal (MaTecK GmbH) was cleaned according to standard procedures [31,32], which involved heating in an $\mathrm{O}_{2}$ atmosphere in order to desorb segregated carbon and flashing at $2300 \mathrm{~K}$ in UHV. The concentration of surface contaminants was below the sensitivity of our X-ray photoemission spectroscopy (XPS) characterization. A high quality of the tungsten surface was inferred from a sharp $(1 \times 1)$ LEED pattern and from the detection of surface related features with PES (see Ref. [33]). Electron-beam heating was used to sublimate $\mathrm{Ni}$ and Fe from high-purity $(5 \mathrm{~N})$ rods. The $\mathrm{Ni}$ and $\mathrm{Fe}$ deposition rates were previously calibrated using an oscillating quartz microbalance and were kept below $2.0 \mathrm{ML} / \mathrm{min}, 1 \mathrm{ML}$ being a single atomic layer referred to the fcc (111) surface, with a thickness of 2.03 and $2.19 \AA$ for Ni and Fe, respectively [2]. Following the literature [34], we applied a two-step procedure for the Ni growth: (i) the $\mathrm{W}$ crystal was kept at $570 \mathrm{~K}$ during the growth of the first Ni monolayer in order to favor the $\mathrm{Ni}$ atom mobility on the substrate and obtain a flat morphology; (ii) the substrate was then kept at room temperature (RT) for further $\mathrm{Ni}$ and $\mathrm{Fe}$ depositions. During the growth of the metallic films the pressure in the UHV chamber remained in the $10^{-8} \mathrm{~Pa}$ range. After the growth, each sample was analyzed by LEED and XPS at RT. Samples were magnetized in situ (either at RT or at cryogenic temperature, down to $30 \mathrm{~K}$ ) by applying a pulsed magnetic field of $2200 \mathrm{Oe}$ along the [001] direction of the $\mathrm{W}$ substrate and analyzed at magnetic remanence. The temperature was set by means of a closed-cycle He cryostat accurately calibrated by replacing the sample with a silicon diode sensor. PES was performed by exciting electrons with $\mathrm{He}$ I or He II radiation ( $h v=21.2$ and $40.8 \mathrm{eV}$, respectively) and detecting them by means of a 150-mm hemispherical analyzer (from SPECS GmbH) with an angular resolution of $\pm 1^{\circ}$. Spin-resolved (SR) spectra were acquired by means of a micro-Mott detector [35], mounted at the exit side of the hemispherical analyzer. Typical full width at half maximum (FWHM) energy resolution values are 15 and $120 \mathrm{meV}$ for PES and SRPES, respectively. The data were acquired at cryogenic temperature $(30 \mathrm{~K})$.

\section{B. Ab initio calculations}

We performed $a b$ initio density functional theory (DFT) simulations by taking the generalized gradient approximation to the exchange and correlation functional as proposed by Perdew, Burke, and Ernzerhof (GGA PBE) [36]. The QUANTUM ESPRESSO package [37] was used for the calculations, within plane waves and ultrasoft pseudopotentials. The $\mathrm{Fe}$ pseudopotential was generated starting from scalar-relativistic all-electron atomic calculations and using nonlinear core corrections, and has been extensively studied in our previous works [38-40]. We used a kinetic energy cutoff of $45 \mathrm{Ry}$ for the wave functions and $200 \mathrm{Ry}$ for the effective potential and the charge density. We considered for the bulk bcc Fe lattice a conventional orthorhombic cell with the $x, y$, and $z$ axes oriented along [001], [1ํㅣㄹ, and [110], respectively, and a bulk lattice parameter of $2.83 \AA$. For that cell we have taken a $14 \times 10 \times$ 10 sampling of the Brillouin zone. Additionally, the computed bands have been projected on a complete set of $d$-like atomic orbitals, oriented according to the $x, y, z$ directions of the orthorhombic cell. This unit cell was the starting point to simulate Fe structural deformations as will be detailed in Sec. IV.

\section{RESULTS}

\section{A. Morphological evolution}

All samples were bilayers comprised of a thin Ni "buffer" layer with a nominal thickness of $2 \mathrm{ML}$, grown on a $\mathrm{W}(110)$ substrate, and a Fe layer with variable thickness (ranging from 2 to $40 \mathrm{ML}$ ). Consistent with the literature, we observe for the buffer layer (not shown) a fcc atomic arrangement already from the first monolayer, exposing the $\mathrm{Ni}(111)$ surface. The Ni lattice is aligned to the substrate according to the NW relationship, $\mathrm{W}[001]_{\text {bcc }} \| \mathrm{Ni}[1 \overline{1} 0]_{\mathrm{fcc}}$ and $\mathrm{W}[1 \overline{1} 0]_{\mathrm{bcc}} \| \mathrm{Ni}[11 \overline{2}]_{\mathrm{fcc}}$. The strain of the Ni film is minimal in the $\mathrm{W}[1 \overline{1} 0]$ direction (about 3\%), while in the $\mathrm{W}[001]$ direction the $\mathrm{Ni}$ layer relaxes and a coincidence structure is 


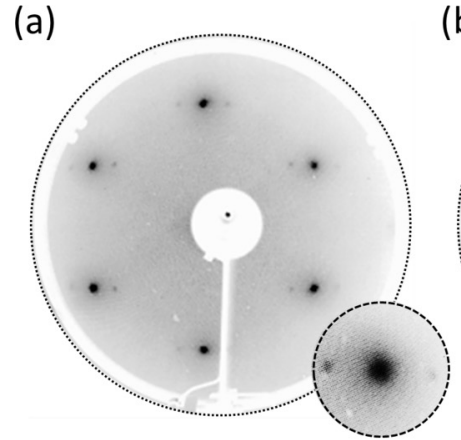

$2 \mathrm{MLFe}$

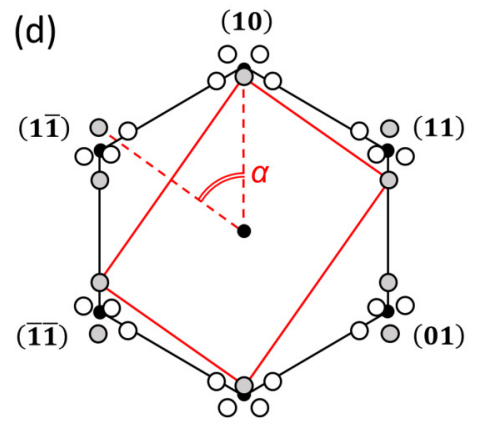

(11) (b)

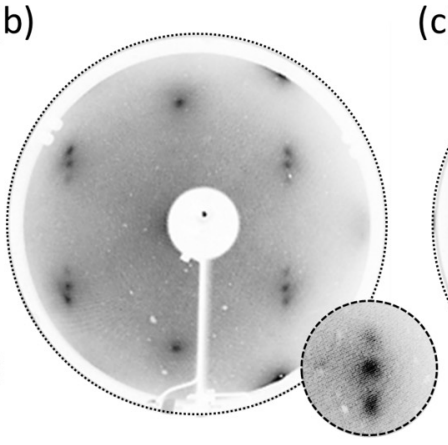

$6 \mathrm{ML} \mathrm{Fe}$

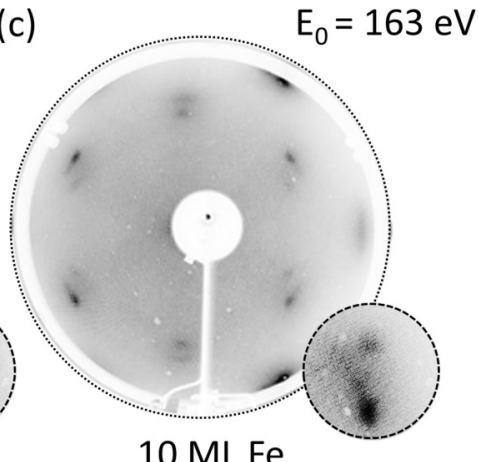

$10 \mathrm{ML} \mathrm{Fe}$<smiles>CC(C)(C)O</smiles>

KS relations:

○ $[\overline{1} 11]_{\mathrm{bcc}} \|[1 \overline{1} 0]_{\mathrm{fcc}}$

○ $[\overline{1} 11]_{\mathrm{bcc}} \|[\overline{1} 01]_{\mathrm{fcc}}$ $[\overline{1} 11]_{\mathrm{bcc}} \|[01 \overline{1}]_{\mathrm{fcc}}$

FIG. 1. LEED patterns for increasing Fe coverages on 2-ML Ni/W(110). The electron beam energy is 163 eV. In the insets: closeup of the diffraction pattern observed near the (11) fcc spot. The beam energy selected for the insets is $125 \mathrm{eV}$. The schematic of panel (d) shows the LEED pattern expected from a bcc surface reconstructed according to the KS relation with white and gray dots, superimposed on the fcc pattern of $\mathrm{Ni}(111)$ (black dots). Gray dots single out only two of the six equivalent KS domains, thus reproducing the experimental pattern of panel (b). The red rectangle shows the bcc Fe(110) primitive cell, rotated by $\alpha=54.8^{\circ}$ with respect to the W[1 10$]$ direction.

formed, where nine Ni cells are matched to seven $\mathrm{W}$ cells. The coincidence structure (also called the $7 \times 1$ structure) gives rise to a series of bright LEED satellite spots aligned in the $\mathrm{W}$ [001] direction. These results, concerning the Ni/W(110) substrate, are in nice agreement with the available literature and have been discussed in Ref. [41].

In the following, we will focus on the growth of the Fe film. Figure 1 shows some characteristic LEED patterns acquired at selected $\mathrm{Fe}$ coverages. For coverages ranging from submonolayer up to about 4 ML [Fig. 1(a)], we observed a hexagonal pattern, practically coincident with the $\mathrm{Ni}(111)$ one. Faint extra spots, related to the underlying $7 \times 1$ coincidence structure, are still visible at the early stages of Fe growth [see, e.g., the inset of Fig. 1(a)]. For coverages larger than about 4 ML [Fig. 1(b)], a different pattern is found, characterized by diffraction spots aligned along the $\mathrm{W}[1 \overline{1} 0]$ direction. This pattern is due to the superposition of the diffraction signal from the fcc layers and a bcc (110) surface, aligned according to the KS relation. As shown in the schematics of Fig. 1(d), a total of six equivalent $\mathrm{KS}$ domains are expected allowing for a nearly perfect lattice matching (within less than 1\%) along the $\langle 1 \overline{1} 0\rangle_{\text {fcc }}$ and $\langle\overline{1} 11\rangle_{\text {bcc }}$ directions. Due to the strain loaded into the $\mathrm{Ni}$ substrate, only two of those domains are stabilized at low Fe coverages, with the bcc Fe(110) unit cell tilted with respect to the W(110) surface lattice. Consistent with the findings of Ref. [2], the angle $\alpha$ is seen to slightly deviate from the value expected from bulk Fe $\left(54.8^{\circ}\right)$, being, for instance, about $56 \pm 1^{\circ}$ for the 6-ML sample of Fig. 1(b).
At larger coverages $(\geqslant 8 \mathrm{ML})$, the LEED pattern appears blurred and more symmetric with respect to threefold rotations around the W[110] axis. The diffraction spots also change from round to crescent shaped [see, e.g., the inset of Fig. 1(c)]. Our interpretation is that now all KS domains are observed, although still with unequal intensity. This KS-like pattern persists up to $40 \mathrm{Fe} \mathrm{ML}$, i.e., the largest film thickness explored in the present study. Good agreement is found with the previous LEED and Auger study of Ref. [2] where, however, the formation of a single-domain NW reconstruction is reported for thicker Fe layers. ${ }^{1}$ We attribute such different behavior to the slightly lower substrate temperature (RT vs $350 \mathrm{~K}$ ), and possibly to slight differences in the preparation and/or thickness of the buffer layer. It should be stressed, however, that the differences between the LEED patterns related to the KS and the NW superstructures can be hardly distinguished in the case of blurred diffraction spots. The simultaneous presence of KS and NW domains at large Fe coverages (reported, for instance, also for the growth of $\mathrm{Fe}$ on $\mathrm{Au}(111)$ [42]) can be neither confirmed nor ruled out from our data. If thicker Ni buffer layers are used, we clearly observe the formation of threefold symmetric NW domains even at small Fe coverages (not shown), in agreement with the available literature on the Fe growth on bulk Ni(111) [43,44].

\footnotetext{
${ }^{1}$ The NW pattern described in Ref. [2] is retrieved by rotating the red rectangle of Fig. 1(d) in order to reach the condition $\alpha=0^{\circ}$.
} 


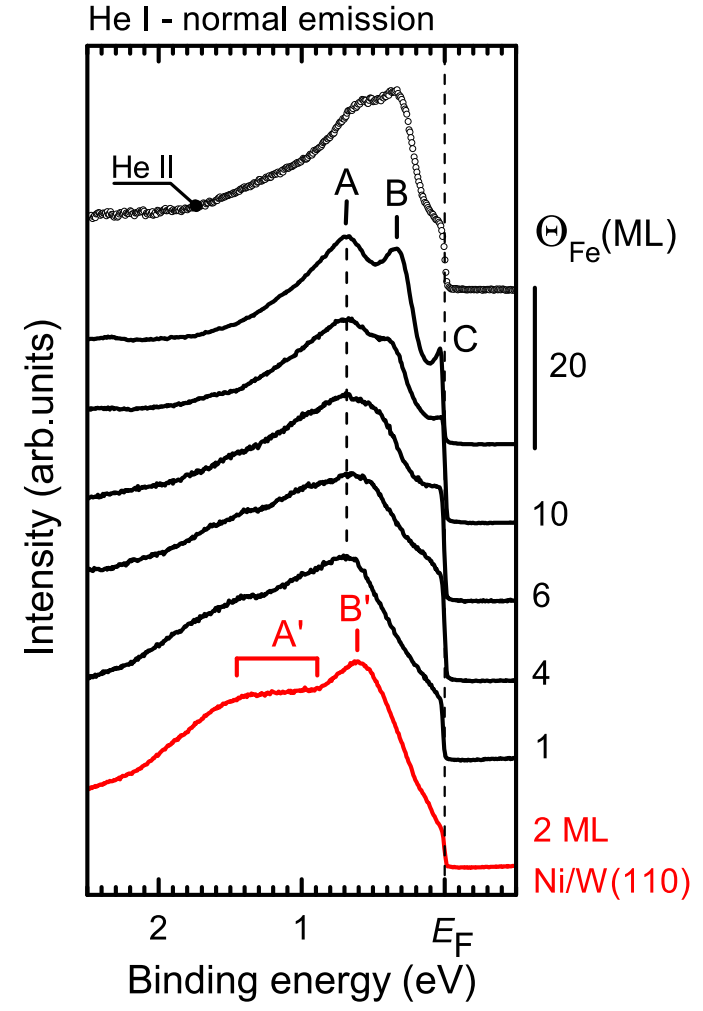

FIG. 2. Photoemission spectra for the 2-ML Ni/W(110) substrate (red line) and for selected Fe coverages $\left(\Theta_{\mathrm{Fe}}\right.$, black lines). The spectra were acquired at normal photoelectron emission, with the substrate kept at $30 \mathrm{~K}$. He I radiation was used for all but the top spectrum (open dots), acquired with He II radiation. Source satellites were subtracted from the He I spectra. Capital letters mark the position of the relevant features discussed in the text: $\mathrm{A}^{\prime}(1-1.4 \mathrm{eV}), \mathrm{B}^{\prime}(0.6 \mathrm{eV}), \mathrm{A}(0.7 \mathrm{eV})$, $\mathrm{B}(0.3 \mathrm{eV})$, and $\mathrm{C}$ close to $E_{\mathrm{F}}$.

\section{B. Electronic structure evolution}

Figure 2 shows a series of spin-integrated spectra taken at normal photoelectron emission for increasing Fe coverage. A substrate temperature of about $30 \mathrm{~K}$ was chosen for our characterization, resulting in sharper line shapes close to the Fermi energy $\left(E_{\mathrm{F}}\right)$. A small reduction to the overall spectral linewidth is also expected at larger binding energy (BE) from the reduced phonon scattering $[45] .{ }^{2}$ Photoemission from the $\mathrm{Ni}$ film is characterized by a peak $\left(\mathrm{B}^{\prime}\right)$ and a shoulder $\left(\mathrm{A}^{\prime}\right)$, the latter partially overlapping with the features related to photoemission from $\mathrm{W} 4 f$ orbitals at a binding energy of about $1.7 \mathrm{eV}$ (see Ref. [41] for further details). The 2-ML Fe spectrum is similar to the $\mathrm{Ni}$ one, excepted for a slight shift of about $0.1 \mathrm{eV}$ of the main photoemission feature, labeled $\mathrm{A}$, that we assign to photoemission from the $\mathrm{Fe}$ electronic states. At larger coverages, a continuous evolution is observed, with the development of peak B at smaller BE and a general sharpening of the photoemission line shapes. Feature $\mathrm{C}$ is related to photoemission from an electronic state located at or slightly below $E_{\mathrm{F}}$, which proved itself to be resilient to surface

\footnotetext{
${ }^{2} \mathrm{~A}$ contribution of about $50 \mathrm{meV}$ FWHM is expected at RT for Fe, according to the formula of Ref. [65].
}

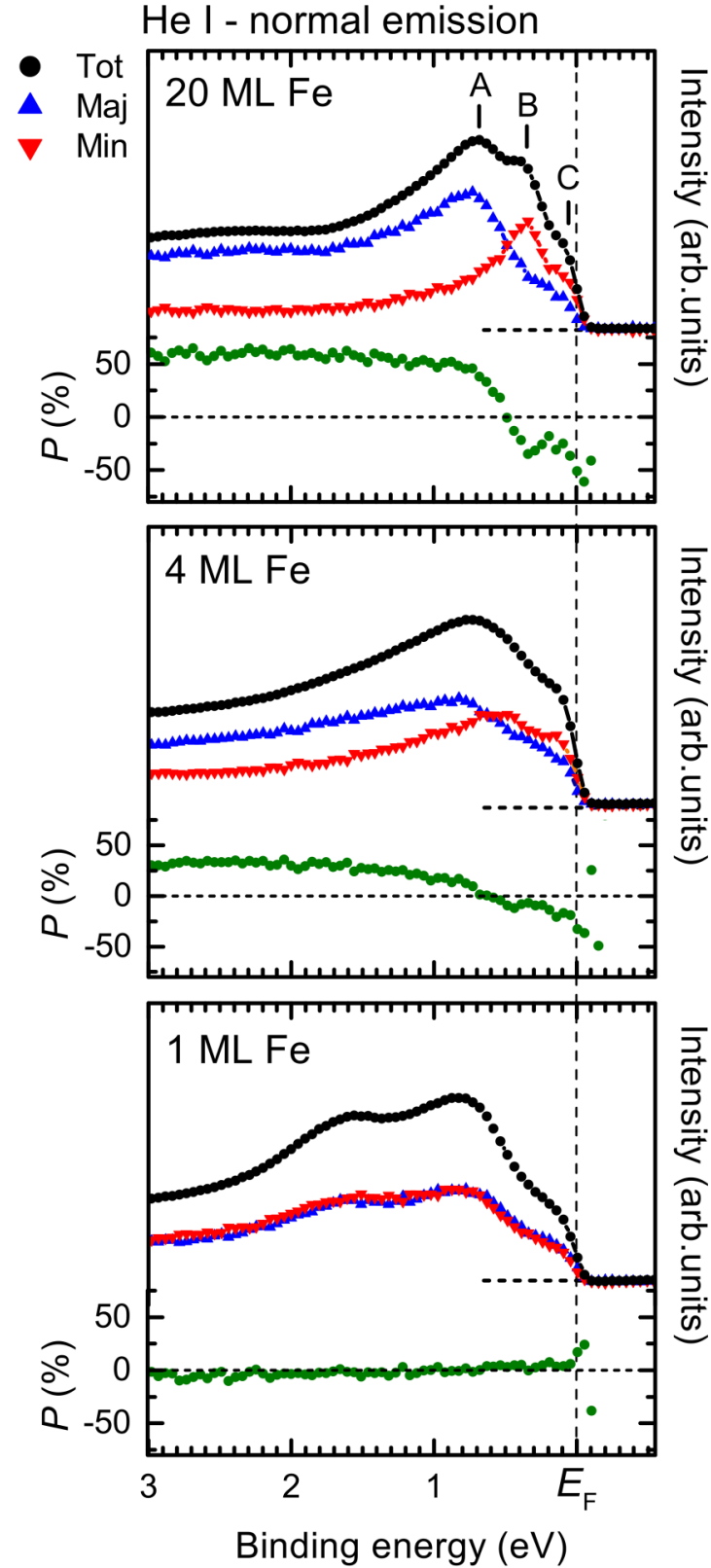

FIG. 3. Spin-resolved photoemission spectra for selected $\mathrm{Fe}$ coverages on 2-ML Ni/W(110). Black dots: spin-integrated spectra. Blue and red triangles: photoemission signal $I^{\uparrow \downarrow}$ from majority and minority (with respect to the magnetization direction) electrons. Green dots: degree of the photoelectrons' spin polarization $P=\left(I^{\uparrow}-I^{\downarrow}\right) /\left(I^{\uparrow}+I^{\downarrow}\right)$, retrieved by correcting the measured spin asymmetry by the Sherman function of the detector $(S=0.14$, according to our previous calibration [30]). Two sets of spectra were acquired at normal photoelectron emission, with the substrate kept at $30 \mathrm{~K}$ and magnetized along two opposite directions, in order to cancel out any instrumental asymmetry $[30,46]$. He I radiation was used and source satellites subtracted.

modification by oxygen (up to $4 \mathrm{~L}$ of $\mathrm{O}_{2}$ dosed at RT, where $1 \mathrm{~L}=10^{-6}$ Torr $\times \mathrm{s}$ ). Furthermore, its intensity is strongly modified by changing the excitation energy from He I to He II (open dots in Fig. 2), i.e., by changing the region in the bulk 

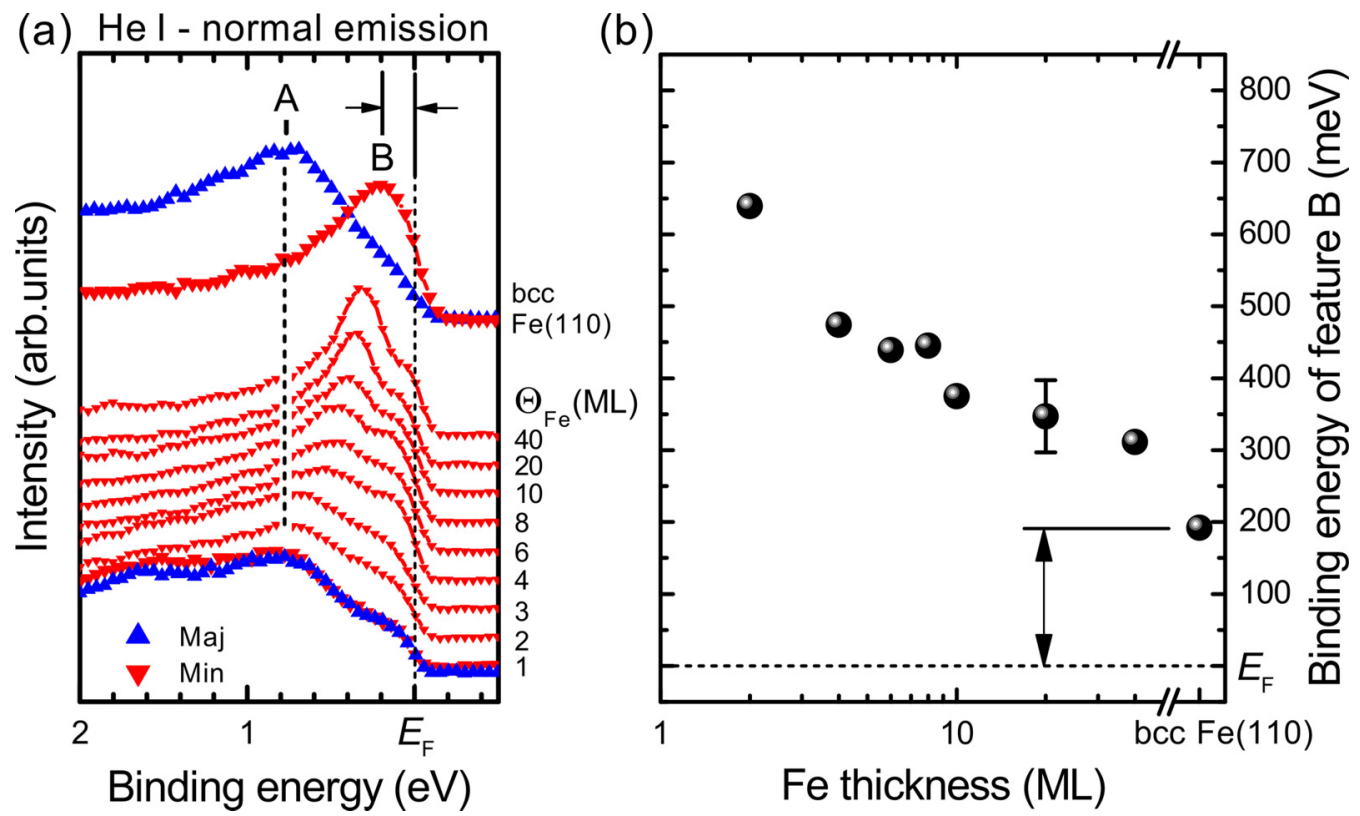

FIG. 4. (a) Spin-resolved photoemission spectra (minority channel) for increasing Fe coverage $\left(\Theta_{\mathrm{Fe}}\right)$, together with the spectra acquired on a reference bcc $\mathrm{Fe}(110)$ surface at RT. He I radiation was used and source satellites subtracted. (b) BE position of feature B as a function of the Fe thickness (displayed on a logarithmic scale).

Brillouin zone (BZ) probed by photoemission. ${ }^{3}$ It is therefore reasonable to assume that feature $\mathrm{C}$ has a bulk character.

Figure 3 shows a series of spin-resolved spectra representative of the magnetic behavior of the Fe films. According to our previous results [41] and the available literature [29], a not-negligible spin polarization is observed, even at RT, for the 2-ML Ni/W(110) buffer layer. The samples were magnetized and characterized with photoemission at cryogenic temperature. No differences were observed with respect to similar measurements performed with the samples magnetized at RT. Referring to the labels introduced for the spin-integrated spectra (see Figs. 2 and 3, black dots), peak A is related to photoemission from majority states (with respect to the magnetization direction), and peak B to photoemission from the minority ones. Despite the lower-energy resolution of the spin-resolved measurements, a faint feature is visible in the minority spectra close to $E_{\mathrm{F}}$, i.e., at about the same $\mathrm{BE}$ of peak $\mathrm{C}$, hinting at a minority character of this spectroscopic feature. The photoelectrons' polarization $P$ (green dots in Fig. 3 ) is positive at large $\mathrm{BE}$ and reverses its sign at about $650 \mathrm{meV}$ for the 4-ML sample and at slightly lower BE (by about $150 \mathrm{meV}$ ) for the 20-ML one. Close to $E_{\mathrm{F}}$, the presence of peaks B and $\mathrm{C}$ is signaled by negative dips, consistent with the minority character of these features. Since most of the spectral evolution seen in Figs. 2 and 3 is related to the development of feature $\mathrm{B}$, we tracked down its position on a larger data set [shown in

\footnotetext{
${ }^{3}$ The electron spectrometer and the measurement conditions were adjusted (for instance, the same pass energy of $0.5 \mathrm{eV}$ was used) in order to keep the same energy resolution for the He I and He II spectra. We also verified that the larger linewidth of the He II source does not compromise the visibility of feature $\mathrm{C}$ by convolving the $\mathrm{He}$ I spectra with a Gaussian line shape of $20 \mathrm{meV}$ FWHM.
}

Fig. 4(a)]. The results of our estimate are plotted in Fig. 4(b) with a logarithmic scale.

In Fig. 4(a), the photoemission spectra related to the minority spin channel are stacked starting from the one related to the 1-ML film (at the bottom of the stack) up to the one related to the 40-ML film (at the top). The position of peak $\mathrm{B}$ could not be determined for the monolayer film, consistent with the absence of any detectable polarization (Fig. 3, bottom panel). The PES spectra from the bcc $\mathrm{Fe}(110)$ surface are added as a reference. The reference sample was grown on $\mathrm{W}(110)$ according to the procedure explained in Ref. [30]. The reference spectra clearly bear some resemblance to the ones reported in Figs. 3 and 4(a): while the BE of feature A is practically coincident with that of the spectra taken from the thin films [the vertical dashed line in Fig. 4(a)], the BE position of peak B can be considered as the asymptotic limit of the evolution observed in Fig. 4(a).

Interestingly, we detect a spin-polarized signal even before the onset of a clear surface reconstruction with LEED. Following the introduction to the present work, a question thus arises as to whether the signal we observe is related to the fcc or rather to the bcc phase. In order to answer this question, we exploited the possibility of our SRPES setup [30] to measure, at normal electron emission and on independent channels, the spin polarization along two orthogonal directions, i.e., in the present case, the $\mathrm{W}[001]$ and $\mathrm{W}[1 \overline{1} 0]$ ones within the W(110) plane. Figure 5 shows the electron polarization in the $\mathrm{W}(110)$ plane for selected photoemission spectra (each dot represents the electron polarization at a specific $\mathrm{BE})$. The preferential scattering of the experimental points along a particular direction is indicative of the direction of the sample magnetization. We were able to reproducibly magnetize the thick ( $\geqslant 4 \mathrm{ML}$ ) Fe films along the W[001] direction, i.e., the direction of the applied magnetic field. At 


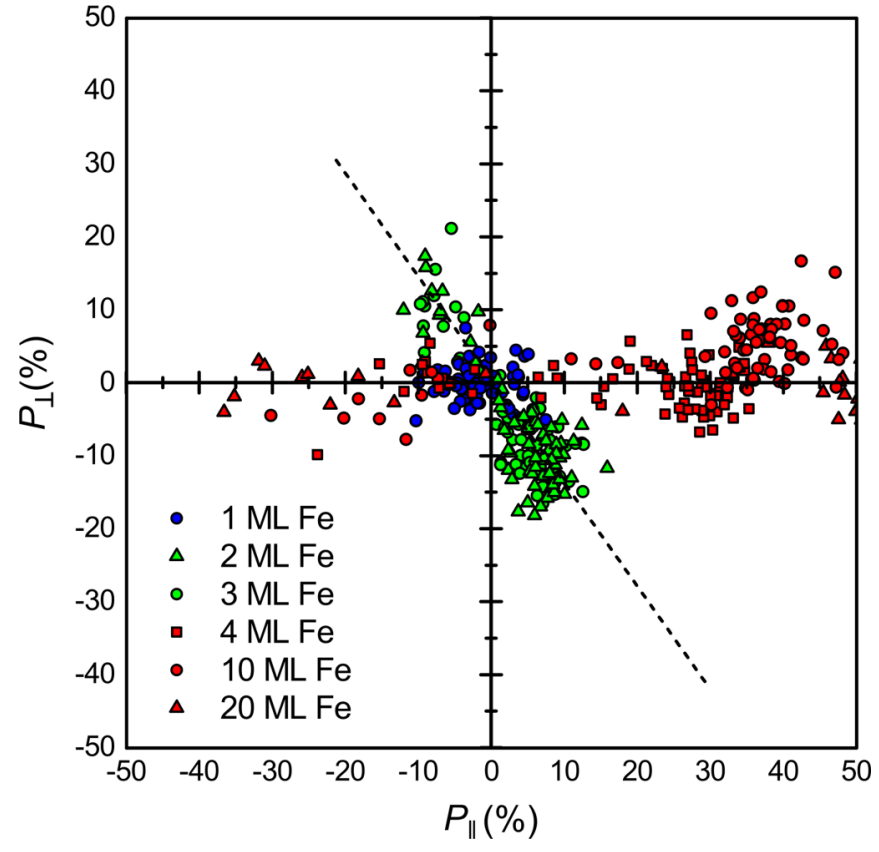

FIG. 5. Photoelectron polarization in the W(110) plane for selected $\mathrm{Fe}$ coverages on 2-ML Ni/W(110), computed according to the formula reported in Fig. 3, i.e., by taking the difference of the spin-up and spin-down intensities $I^{\uparrow \downarrow}$, normalized by their sum. Each data point represents the electron polarization measured, at a given binding energy, along the main axis of our apparatus $\left(P_{\|}\right)$ and perpendicular to it $\left(P_{\perp}\right)$. These two directions correspond to the $\mathrm{W}$ [001] and [1ํㅣㄹ directions at normal electron emission, respectively. Samples were magnetized along the W[001] direction and measured by using He I radiation in magnetic remanence. The electron polarization is evaluated from the spectra acquired in the energy range spanning from $3 \mathrm{eV}$ to $E_{\mathrm{F}}$. Following Refs. [30,46], the instrumental asymmetries have been eliminated from the data by repeating each spin-resolved measurement with the sample magnetized along the opposite direction.

lower coverages, still characterized by a $(1 \times 1)$ LEED pattern [Fig. 1(a)], a non-negligible spin polarization is observed and the magnetization axis is tilted by about $55^{\circ}$ with respect to the $\mathrm{W}[001]$ direction, which corresponds also to the direction of the easy magnetization axis of the Ni substrate [41]. Following our previous discussion about the morphological evolution of thin Fe films, it is easy to recognize that the magnetization direction for low coverages is actually coincident with one of the two equivalent $\langle 001\rangle_{\text {bcc }}$ directions of the KS reconstruction of Fig. 1(b). We stress that the observation of few KS variants and, therefore, of a tilted magnetization direction in the $\mathrm{Fe} / \mathrm{Ni}$ system is made possible, in the present case, by the anisotropic stress loaded in the Ni film from the W(110) substrate, as highlighted in the Introduction. We can thus relate the magnetic signal detected at low coverages to the formation of bcc-like phases. As long as thicker films are concerned, it is worth recalling that we could not univocally determine the alignment of the bcc Fe lattice with respect to the substrate. In those cases where the growth of different crystallographic domains is observed, no in-plane easy magnetization axes are found and the film cannot retain its full magnetization at remanence. This results in a reduced polarization of the photoemission spectra which show, for instance, the same features (with different intensities) in both spin channels [15]. At variance with this picture, Figs. 3(a) and 3(b) show fully resolved peaks, hinting at a preferential magnetization of $\mathrm{Fe}$ along the W[001] direction, which is indeed coincident with one of the easy magnetization axes of bcc $\mathrm{Fe}(110)$ films [47], if we assume that they are aligned as the $\mathrm{W}$ substrate.

\section{DISCUSSION}

The experimental results of the previous section provide a rather detailed picture of the electronic and magnetic evolution of thin Fe films. At low Fe coverages, a pseudomorphic fcc film is formed. The absence of any in-plane magnetic signal for the 1-ML Fe film can be related either to the presence of a nonmagnetic or "dead" $\mathrm{Fe}$ layer, or to the formation of a ferromagnetic film characterized by an out-of-plane magnetization. Both results would be fully consistent with the literature $[34,48]$ on the existence of a spin-reorientation transition (i.e., a rotation of the sample magnetization from an in-plane to an out-of-plane direction) in Fe/Ni bilayers with a $\mathrm{Ni}$ thickness ranging from two up to ten layers and for a $\mathrm{Fe}$ coverage up to a single layer. An out-of-plane magnetization with a Curie temperature $\left(T_{C}\right)$ of about $250 \mathrm{~K}$, for instance, has been reported in Ref. [34] for the system comprised by a Fe monolayer grown on a 2-ML Ni/W(110) substrate. As a third alternative, the $\mathrm{Fe}$ film might be ferromagnetic with a very small $T_{C}$. This hypothesis, however, is rather unlikely, given that (i) $T_{C}$ well above the lowest temperature we used (30 K) are reported for Fe coverages close to the ML completion in the $\mathrm{Fe} / \mathrm{W}(110)$ case [49]; (ii) the proximity with the ferromagnetic $\mathrm{Ni}$ substrate should promote either a ferromagnetic or an antiferromagnetic ordering of a magnetic overlayer, as in the case of ML-range $\mathrm{Cr}$ films on $\mathrm{Fe}(001)$ [50]. ${ }^{4}$ At larger $\mathrm{Fe}$ coverages, we start to observe the spectroscopic signatures of the bcc phase. Interestingly, the changes we report for the electronic structure are not matched by a simultaneous evolution of the LEED patterns (although related to the very same samples). This discrepancy, which might be at the roots of the past difficulties in understanding the electronic structure of thin Fe films, is probably related to (i) the slightly larger information depth [51] that characterizes the LEED technique (which is therefore more sensitive to the fcc layer), and (ii) the actual size and location of the bcc nuclei, which might develop at the edges of the islands formed during the growth of the Fe film [3], and might be therefore characterized by a lower degree of crystallographic order. Similar results are reported for the growth of $\mathrm{Fe}$ on $\mathrm{Au}(111)$ in Ref. [15], where bcc crystallites are detected with STM after the completion of the first Fe layer, about $1 \mathrm{ML}$ before the appearance of satellite

\footnotetext{
${ }^{4}$ We note that, although it is in principle possible to assess the existence of an out-of-plane electron polarization by simply tilting the sample, we could not obtain any clear evidence of such a phenomenon within the rather high uncertainty of about $5 \%$ characterizing this kind of experiment. This is mainly due to the impossibility, with our current apparatus, of reversing the out-of-plane sample magnetization in a controlled way in order to cancel out instrumental asymmetries, as needed to unambiguously detect small polarization values.
} 
(a)

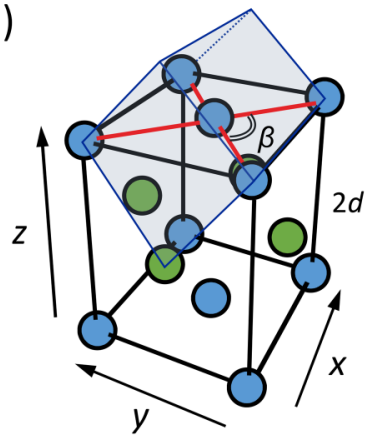

(b)

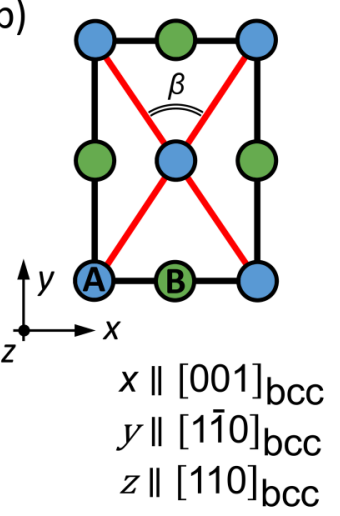

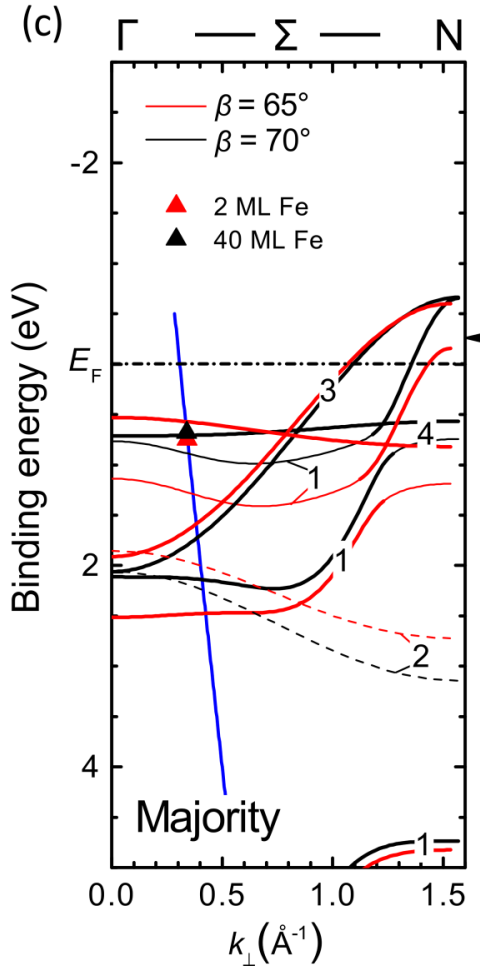

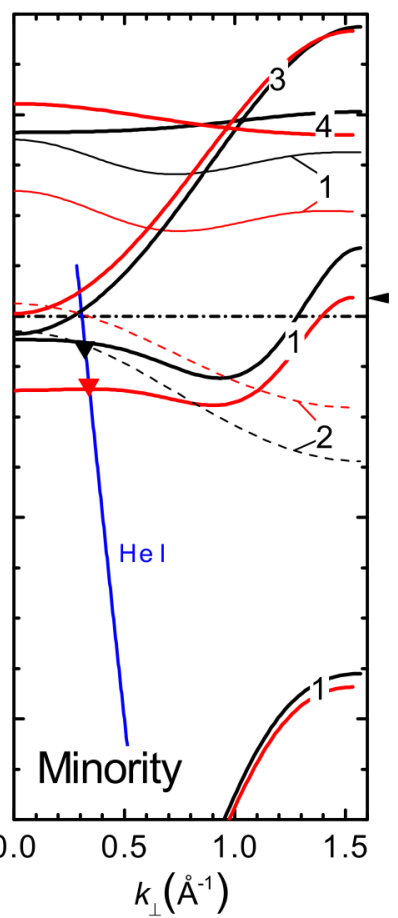

FIG. 6. (a) Sketch of the primitive orthorhombic cell used to simulate the deformation of the Fe lattice. A wireframe is added (thin blue lines), representative of the primitive bcc cell structure. (b) Atomic stacking in the direction perpendicular to the surface for the bcc lattice [letters A and B identify Fe atoms on different (110) bcc planes]. A set of relations mapping the Cartesian coordinate system used in the simulation onto the crystallographic directions of the bcc lattice is also shown. (c) Spin-resolved band structure computed along the $\Gamma \mathrm{N}$ direction of the bcc Brillouin zone. The bands are labeled according to the standard symmetry group notation for the (110) $)_{\text {bcc }}$ surface [53]. Dashed lines highlight $\Sigma_{2}$ bands. The states with a $d_{x^{2}-y^{2}}$ or $d_{x y}$ character are drawn with thin lines. As explained in the main text, the simulated bands have been rigidly shifted upwards; the original position of $E_{\mathrm{F}}$ can be retrieved from the small arrows placed along the right axes. Blue lines: BE vs internal momentum $k_{\perp}$ for photoelectrons excited by He I radiation. Symbols: experimental position of features A (majority spins, up triangles) and B (minority spins, down triangles) for the 2- and 40-ML Fe films.

spots in LEED. Another example is related to the growth of ultrathin Fe films on $\mathrm{Cu}(111)$ by pulsed laser deposition, where a hexagonal $p(1 \times 1)$ pattern is observed with LEED up to a thickness of $6 \mathrm{ML}$ [19], i.e., well after the formation of the bcc nuclei starting from the second Fe layer (see, for instance, the STM study of Ref. [52]). From the point of view of our magnetic characterization, the presence of such a small amount of bcc Fe develops a sufficiently strong magnetic anisotropy term turning the remanent magnetization in the film plane.

We have described in the previous section the modifications occurring in the PES spectra as a function of the Fe thickness. In particular, we focused on the evolution of the minority spin signal, characterized by the development of peak B. The observed evolution is largely independent of the measurement temperature: for a given coverage, no clear changes in the energy position nor in the intensities of the majority and minority features were observed in spin-resolved spectra. This result is particularly noteworthy since similar modifications might be in principle related to variations in the macroscopic magnetization of the sample. Following the discussion of the previous section, we can therefore assume that the films under investigation were magnetically almost saturated and conclude that the observed evolution is linked to the structural modifications occurring within the bcc phase. Simulating the impact of the fcc to bcc structural transition on the electronic band structure is a formidable task that cannot be tackled directly given the complexity of the (largely unknown) precise atomistic configuration. According to the literature, the "martensitic front," i.e., the interface between the fcc and bcc phases, is formed at low Fe coverages and then buried under the subsequent Fe layers, consistent with the fact that the habit plane of the martensitic transition is actually coincident with a $\{111\}_{\text {fcc }}$ plane [2]. The abrupt change of the lattice unit cell expected at the bcc/fcc interface is therefore likely followed by a more gradual relaxation with increasing distance from the interface. The authors of Ref. [43] proposed the following atomic displacements in order to explain their X-ray photoelectron diffraction (XPD) results for the $\mathrm{Fe} / \mathrm{Ni}(111)$ system: for increasing Fe coverages, the deformation of the Fe unit cell in the $(110)_{\text {bcc }}$ plane is matched to a concomitant variation of the spacing $d$ between the $(110)_{\text {bcc }}$ planes. A value of $2.11 \AA$ was found for a 6-ML Fe film, corresponding to a $+3.9 \%$ expansion with respect to the bulk value. ${ }^{5}$ A similar

\footnotetext{
${ }^{5}$ Lattice distortions are detected by XPD even for very thin Fe films in Ref. [43]. However, a quantitative analysis is provided only for thicker films.
} 
evolution, experimentally investigated with LEED only for in-plane deformations, was observed by the authors of Ref. [2] in thin Fe films grown on 2-ML Ni/W(110). Finally, distorted bcc Fe phases (called KS-3 and KS-7 phases) have been detected even in 2- or 3-ML Fe films on the analogous system $\mathrm{Fe} / \mathrm{Cu}(111)[3]$.

We thus considered a simple model in which the electronic structure of multilayer Fe films can be interpreted in terms of bulk Fe bcc crystal with distorted geometry [2,43], neglecting surface and interface effects. The pristine lattice is described by the orthorhombic cell shown in Fig. 6(a), where the $z$ axis is oriented along the [110] direction and the $x y$ plane contains the rectangular base cell corresponding to the $(110)_{\mathrm{bcc}}$ surface. The deformation of the Fe lattice in the $x y$ plane is simulated by decreasing the $\beta$ angle [Fig. 6(a)] from its value in the relaxed $(110)_{\text {bcc }}$ surface $\left(70.5^{\circ}\right)$ down to $65^{\circ}$, chosen on the basis of the experimental results of Ref. [2]. Considering the nearly perfect matching between the $(110)_{\text {bcc }}$ and the (111) fcc diagonals [as shown in Fig. 1(d)] their length in the $x y$ rectangle [red lines in Figs. 6(a) and 6(b)] is kept fixed at the characteristic value of the relaxed bcc lattice $\left(\sqrt{3} a_{\text {bcc }}\right)$ during the $\beta$ variation. The interlayer distance $d(\beta)$ was obtained by geometry optimization. For the deformed lattice $\left(\beta=65^{\circ}\right)$, we found a value of $d\left(65^{\circ}\right)$ which is larger (by $0.04 \AA$ ) than the optimized spacing $d\left(70.5^{\circ}\right)=2.01 \AA$ for the relaxed cell, in nice agreement with the experimental study of Ref. [43]. The band structure for $\beta=70.5^{\circ}$ and $\beta=65^{\circ}$ is reported in Fig. 6(c) for the $[110]_{\text {bcc }}$ direction, perpendicular to the surface (wave vector $k_{\perp}$ along the $\Gamma-\Sigma-\mathrm{N}$ line in reciprocal space) and probed with our system at normal electron emission $\left(k_{\|}=\bar{\Gamma}\right)$. The results for $\beta=70.5^{\circ}$ are in good agreement with the available literature on $a b$ initio calculations on bulk Fe [54-56]. Slight discrepancies do exist with the experimental position of the photoemission features, generally located at lower BE, which have been nevertheless well documented [57] and can be partially mended by employing more sophisticated computational schemes, such as the one-step model for photoemission, and improving the description of correlation effects [53]. In order to facilitate the comparison between our experimental data and the simulations, we therefore decided to apply a rigid upward shift of 260 (180) $\mathrm{meV}$ for the majority (minority) states, consistent with literature results (Fig. 4(a) in Ref. [53]) related to the $\Gamma$ point. Figure 6(c) clearly shows that good agreement is obtained between the BE position of the photoemission features and the simulated band structure after the BE scale modification. The bands have been labeled according to the irreducible representations of the $C_{2 v}$ group, pertinent to the $\Sigma$ direction [58]. Each band exhibits a smooth dependence on $\beta$, which is different according to the state involved. For example, three nearly degenerate minority bands are found around $\Gamma$ at a BE of $0.2 \mathrm{eV}$ when $\beta=70.5^{\circ}$. In passing to $\beta=65^{\circ}$, two of such states are shifted towards $E_{\mathrm{F}}$, namely, the one labeled 3 (with $d_{z x}$ character) and that labeled 2 (with $d_{x y}$ character at the $\Gamma$ point), whereas the band labeled 1 (with $d_{z^{2}}$ character) shifts to higher BE exhibiting the largest variation. For the majority states observed within about $1 \mathrm{eV}$ from $E_{\mathrm{F}}$, band 1 shifts towards larger BE, whereas band 4 is more or less stationary. The orbital character at the $\Gamma$ point of bands 1 and 4 is $d_{x^{2}-y^{2}}$ and $d_{z y}$, respectively. In order to compare our experimental results with the simulated band structure of Fig. 6(c), we estimated the photoelectrons' internal momentum $\left(k_{\perp}\right)$ following the method outlined in Ref. [59] by assuming a free-electron-like final state in a constant inner potential $V_{0}$ of $14.8 \mathrm{eV}$ and a work function of $5 \mathrm{eV}$ [53]. The blue lines in Fig. 6(c) show the internal momentum associated with electrons excited with $\mathrm{He}$ I radiation and allow to pinpoint the position of photoemission features $\mathrm{A}$ and $\mathrm{B}$ in reciprocal space for very thin (2-ML) and thick (40-ML) $\mathrm{Fe}$ films. By referring to our labeling convention, feature A (B) accounts for photoemission from the majority $\Sigma_{1,4}^{\uparrow}$ (minority $\Sigma_{1}^{\downarrow}$ ) bands [53], while feature C close to $E_{\mathrm{F}}$ (not shown) is related to photoemission from the minority $\Sigma_{3}^{\downarrow}$ band. No emission is expected from $\Sigma_{2}$ bands [dashed lines in Fig. 6(c)], which are dipole forbidden at normal emission [58], nor has it ever been observed [60]. The $\Sigma_{1,4}^{\uparrow}$ and $\Sigma_{1}^{\downarrow}$ bands show almost no energy dispersion in the $\Gamma \mathrm{N}$ direction, while the $\Sigma_{3}^{\downarrow}$ band is seen to rise steeply in energy and cross $E_{\mathrm{F}}$ away from the $\Gamma$ point. Feature B in the minority spectra shifts toward $E_{\mathrm{F}}$ as the thickness of the Fe layer is increased; similarly, band $\Sigma_{1}^{\downarrow}$ moves close to its bulk position at lower BE as the deformation of the orthorhombic Fe cell is reduced, which is consistent with our prediction of a gradual relaxation of the Fe crystal structure away from the fcc/bcc interface. Feature A results from the superposition of the photoemission signal from bands $\Sigma_{1,4}^{\uparrow}$, which starts from different BE positions for $\beta=65^{\circ}$ and they merge together in bulk bcc Fe. This behavior might explain the apparent stationary position of feature $\mathrm{A}$ in the experiment, and the overall larger width of the majority line shape with respect to the minority one (see, e.g., Fig. 3, top spectrum). In addition, we note that a lower photoemission intensity is expected from purely in-plane orbitals $d_{x^{2}-y^{2}}$ and $d_{x y}$, if the actual values of the matrix elements for the related dipole-allowed transitions are considered [61]. Those bands with a prevalent $d_{x^{2}-y^{2}}$ and $d_{x y}$ character are drawn with thinner lines in Fig. 6(c). By inspecting Fig. 6(c) we can therefore conclude that the shifting band $\Sigma_{1}^{\uparrow}$ contributes less than the more stationary $\Sigma_{4}^{\uparrow}$ band to the actual position of feature A. Finally, the $\Sigma_{3}^{\downarrow}$ band is expected to cross $E_{\mathrm{F}}$ as the thickness of the Fe layer is increased (i.e., by increasing $\beta$ towards the bulk value), at about the same wave vector probed with He I radiation. We suggest that this modification is related to the development of peak $\mathrm{C}$, clearly visible in Fig. 2 only for thick Fe films. The dependence upon thickness we observe for the $d_{z^{2}}$ orbital (feature B) is consistent with the $\sim 2.5 \%$ increase of cell size along $z$ when $\beta$ decreases from $70.5^{\circ}$ to $65^{\circ}$, where band narrowing is expected from simple tight binding theory. ${ }^{6}$ A seemingly simple explanation for the evolution of the $d_{z x}$ and $d_{x y}$ states is not trivially identifiable, because of the concomitant variation of the cell sizes along the

\footnotetext{
${ }^{6}$ Considering the downward curvature of the $d_{z^{2}}$ band close to $\Gamma$, band narrowing is expected to shift the band towards larger BE. In order to follow the energy dispersion of the $d_{z^{2}}$ state, one has to notice that it hybridizes with the highly dispersing $s p$ band, forming an avoided crossing halfway between $\Gamma$ and $\mathrm{N}$, so that band $\Sigma_{1}^{\downarrow}$ crossing $E_{F}$ at about $\mathrm{N}$ has a $s p$ character while the $d_{z^{2}}$ state is found at $4 \mathrm{eV} \mathrm{BE}$.
} 
$x(-7 \%)$ and $y$ directions $(+3.5 \%)$ and the mixed orientation of those $d$ orbitals as well as of the nearest-neighbor atoms. The computed magnetic moment per atom is $2.17 \mu_{\mathrm{B}}$, in good agreement with other literature results for bcc Fe $[62,63]$ and independent from the angle $\beta$ in the $65^{\circ}-70.5^{\circ}$ interval. Although an indirect confirmation of this result by looking at the exchange splitting of the photoemission features is impeded in the present case by the broad line shape of the experimental spectra for small Fe coverages and large $\mathrm{BE}$ [the majority spin counterpart for feature B should be located at a $\mathrm{BE}$ of about $2 \mathrm{eV}$, according to Fig. 6(c)], this prediction from $a b$ initio calculations is nevertheless reasonable since (i) the tetragonal deformation of the Fe cell only mildly changes the volume per atom, which is known to directly affect the magnetic moment [6], and (ii) a nearly constant magnetic moment is reported by the authors of Ref. [64] with $\mathrm{x}$-ray magnetic circular dichroism (XMCD) during the fcc to bcc phase transformation for the similar $\mathrm{Fe} / \mathrm{Cu}(111)$ system.

\section{CONCLUSIONS}

The growth of Fe on a 2-ML Ni/W(110) substrate produces, for coverages in the monolayer range, a pseudomorphic fcc layer and no detectable in-plane magnetization. At multilayer coverage, we observe a non-nil spin polarization attributed to the onset of the fcc to bcc martensitic transition with the formation of bcc nuclei showing a KS orientation relationship. A clear restructuring of the Fe surface is visible with LEED only for Fe layers with a thickness larger than $4 \mathrm{ML}$. The electronic structure of the bcc phase is seen to continuously evolve with the $\mathrm{Fe}$ coverage towards the one expected from bulklike Fe; in particular, most of the changes observed with He I radiation occur within the minority states. $A b$ initio calculations, simulating the deformation of the bcc lattice in such a way as to approximate the supposed structure of the metastable bcc phase, are able to reproduce the observed spectral evolution.

\section{ACKNOWLEDGMENTS}

The authors would like to thank A. Picone and F. Bottegoni for fruitful discussions. G.B.'s research is funded by Fondazione Cariplo (Grant No. 2013-0623 SEARCH-IV).
[1] W. Martienssen and H. Warlimont, Springer Handbook of Condensed Matter and Materials Data (Springer Science \& Business Media, New York, 2006).

[2] H. L. Johnston, C. S. Arnold, and D. Venus, Thicknessdependent fcc to bcc structural change in iron films: Use of a 2-ML Ni/W(110) substrate, Phys. Rev. B 55, 13221 (1997).

[3] A. Biedermann, W. Rupp, M. Schmid, and P. Varga, Coexistence of fcc- and bcc-like crystal structures in ultrathin Fe films grown on $\mathrm{Cu}(111)$, Phys. Rev. B 73, 165418 (2006).

[4] Phase Transformations in Materials, edited by G. Kostorz (Wiley-VCH Verlag GmbH \& Co. KGaA, Weinheim, FRG, 2001).

[5] G. Bussetti, M. Riva, A. Picone, A. Brambilla, L. Duò, M. Finazzi, and F. Ciccacci, Martensitic transition during Ni growth on $\mathrm{Fe}(001)$ : evidence of a precursor phase, New J. Phys. 14, 053048 (2012).

[6] V. L. Moruzzi, P. M. Marcus, K. Schwarz, and P. Mohn, Ferromagnetic phases of bcc and fcc Fe, Co, and Ni, Phys. Rev. B 34, 1784 (1986).

[7] D. Bagayoko and J. Callaway, Lattice-parameter dependence of ferromagnetism in bcc and fcc iron, Phys. Rev. B 28, 5419 (1983).

[8] J. C. Boettger, Strain-dependent band-structure effects in the magnetic moment of a hexagonal Fe monolayer, Phys. Rev. B 47, 1138(R) (1993).

[9] Magnetism and Structure in Systems of Reduced Dimension, edited by F. C. Farrow, B. Dieny, M. Donath, A. Fert, and B. D. Hermsmeier (Springer US, Boston, MA, 1993).

[10] F. Ciccacci and S. De Rossi, Empty electronic states in magnetic thin films: $\mathrm{Fe}$ on $\mathrm{Au}(100), \mathrm{Ag}(100)$, and $\mathrm{Cu}(100)$, Phys. Rev. B 51, 11538 (1995).

[11] P. Allongue, F. Maroun, H. F. Jurca, N. Tournerie, G. Savidand, and R. Cortès, Magnetism of electrodeposited ultrathin layers: Challenges and opportunities, Surf. Sci. 603, 1831 (2009).
[12] M. Á. Niño, J. Camarero, L. Gómez, J. Ferrón, J. J. de Miguel, and R. Miranda, Surfactant-assisted epitaxial growth and magnetism of Fe films on $\mathrm{Cu}(111)$, J. Phys.: Condens. Matter 20, 265008 (2008)

[13] S. Andrieu, M. Piecuch, and J. F. Bobo, Fe growth on (0001) hcp $\mathrm{Ru}$ and (111) fcc Ir: Consequences for structural and magnetic properties, Phys. Rev. B 46, 4909 (1992).

[14] K. von Bergmann, S. Heinze, M. Bode, G. Bihlmayer, S. Blügel, and R. Wiesendanger, Complex magnetism of the Fe monolayer on $\operatorname{Ir}(111)$, New J. Phys. 9, 396 (2007).

[15] T. Allmers and M. Donath, Magnetic properties of Fe films on flat and vicinal $\mathrm{Au}(111)$ : Consequences of different growth behavior, Phys. Rev. B 81, 064405 (2010).

[16] L. Hammer, S. Müller, and K. Heinz, $\mathrm{Fe} / \mathrm{Cu}(100)$ - a test case for the understanding of epitaxially grown magnetic thin films, Surf. Sci. 569, 1 (2004).

[17] H. L. Meyerheim, J.-M. Tonnerre, L. Sandratskii, H. C. N. Tolentino, M. Przybylski, Y. Gabi, F. Yildiz, X. L. Fu, E. Bontempi, S. Grenier, and J. Kirschner, New Model for Magnetism in Ultrathin fcc $\mathrm{Fe}$ on $\mathrm{Cu}(001)$, Phys. Rev. Lett. 103, 267202 (2009).

[18] F. Himpsel, Exchange Splitting of Epitaxial fcc $\mathrm{Fe} / \mathrm{Cu}(100)$ versus bcc Fe/Ag(100), Phys. Rev. Lett. 67, 2363 (1991).

[19] J. Shen, P. Ohresser, C. V. Mohan, M. Klaua, J. Barthel, and J. Kirschner, Magnetic Moment of fcc Fe(111) Ultrathin Films by Ultrafast Deposition on Cu(111), Phys. Rev. Lett. 80, 1980 (1998).

[20] A. Biedermann, R. Tscheließnig, M. Schmid, and P. Varga, Crystallographic Structure of Ultrathin Fe Films on $\mathrm{Cu}(100)$, Phys. Rev. Lett. 87, 086103 (2001).

[21] M. Donath, M. Pickel, A. B. Schmidt, and M. Weinelt, Ferromagnetic $\mathrm{Fe}$ on $\mathrm{Cu}(001)$ throughout the fcc-like phase: arguing from the viewpoint of the electronic structure, J. Phys.: Condens. Matter. 21, 134004 (2009). 
[22] T. Burkert, O. Eriksson, S. I. Simak, A. V. Ruban, B. Sanyal, L. Nordström, and J. M. Wills, Magnetic anisotropy of $\mathrm{L}_{0} \mathrm{FePt}$ and $\mathrm{Fe}_{1-x}$ Mn, Phys. Rev. B 71, 134411 (2005).

[23] T. Burkert, L. Nordström, O. Eriksson, and O. Heinonen, Giant Magnetic Anisotropy in Tetragonal FeCo Alloys, Phys. Rev. Lett. 93, 027203 (2004).

[24] A. Moser, K. Takano, D. T. Margulies, M. Albrecht, Y. Sonobe, Y. Ikeda, S. Sun, and E. E. Fullerton, Magnetic recording: advancing into the future, J. Phys. D: Appl. Phys. 35, R157 (2002).

[25] C. Chappert, A. Fert, and F. N. Van Dau, The emergence of spin electronics in data storage, Nat. Mater. 6, 813 (2007).

[26] J. Gloss, S. Shah Zaman, J. Jonner, Z. Novotny, M. Schmid, P. Varga, and M. Urbánek, Ion-beam-induced magnetic and structural phase transformation of Ni-stabilized face-centeredcubic Fe films on $\mathrm{Cu}(100)$, Appl. Phys. Lett. 103, 262405 (2013).

[27] A. Picone, G. Bussetti, M. Riva, A. Calloni, A. Brambilla, L. Duò, F. Ciccacci, and M. Finazzi, Oxygen-assisted Ni growth on $\mathrm{Fe}(001)$ : Observation of an "anti-surfactant" effect, Phys. Rev. B 86, 075465 (2012).

[28] E. Bauer and J. H. van der Merwe, Structure and growth of crystalline superlattices: From monolayer to superlattice, Phys. Rev. B 33, 3657 (1986).

[29] K.-P. Kämper, W. Schmitt, D. A. Wesner, and G. Güntherodt, Thickness dependence of the spin- and angle-resolved photoemission of ultrathin, epitaxial $\mathrm{Ni}(111) / \mathrm{W}(110)$ layers, Appl. Phys. A: Solids Surf. 49, 573 (1989).

[30] G. Berti, A. Calloni, A. Brambilla, G. Bussetti, L. Duò, and F. Ciccacci, Direct observation of spin-resolved full and empty electron states in ferromagnetic surfaces, Rev. Sci. Instrum. 85, 073901 (2014).

[31] C. Kozioł, G. Lilienkamp, and E. Bauer, Electronic properties of ultrathin nickel films on W(110), Phys. Rev. B 41, 3364 (1990).

[32] K. Miyamoto, A. Kimura, K. Kuroda, T. Okuda, K. Shimada, H. Namatame, M. Taniguchi, and M. Donath, Spin-Polarized Dirac-Cone-Like Surface State with $d$ Character at W(110), Phys. Rev. Lett. 108, 066808 (2012).

[33] R. H. Gaylord and S. D. Kevan, Spin-orbit-interaction-induced surface resonance on W(011), Phys. Rev. B 36, 9337(R) (1987).

[34] D. Sander, A. Enders, C. Schmidthals, J. Kirschner, H. L. Johnston, C. S. Arnold, and D. Venus, Structure and perpendicular magnetization of Fe/Ni(111) bilayers on W(110), J. Appl. Phys. 81, 4702 (1997)

[35] F. B. Dunning, Mott electron polarimetry, Nucl. Instrum. Methods Phys. Res., Sect. A 347, 152 (1994).

[36] J. P. Perdew, K. Burke, and M. Ernzerhof, Generalized Gradient Approximation Made Simple, Phys. Rev. Lett. 77, 3865 (1996).

[37] P. Giannozzi, S. Baroni, N. Bonini, M. Calandra, R. Car, C. Cavazzoni, D. Ceresoli, G.L. Chiarotti, M. Cococcioni, I. Dabo, A. Dal Corso, S. de Gironcoli, S. Fabris, G. Fratesi, R. Gebauer, U. Gerstmann, C. Gougoussis, A. Kokalj, M. Lazzeri, L. MartinSamos et al. QUANTUM ESPRESSO: a modular and open-source software project for quantum simulations of materials, J. Phys.: Condens. Matter. 21, 395502 (2009).

[38] A. Picone, G. Fratesi, M. Riva, G. Bussetti, A. Calloni, A. Brambilla, M. I. Trioni, L. Duò, F. Ciccacci, and M. Finazzi,
Self-organized chromium oxide monolayers on $\mathrm{Fe}(001)$, Phys. Rev. B 87, 085403 (2013).

[39] A. Picone, M. Riva, G. Fratesi, A. Brambilla, G. Bussetti, M. Finazzi, L. Duò, and F. Ciccacci, Enhanced Atom Mobility on the Surface of a Metastable Film, Phys. Rev. Lett. 113, 046102 (2014).

[40] M. Riva, A. Picone, D. Giannotti, A. Brambilla, G. Fratesi, G. Bussetti, L. Duò, F. Ciccacci, and M. Finazzi, Mesoscopic organization of cobalt thin films on clean and oxygen-saturated Fe(001) surfaces, Phys. Rev. B 92, 115434 (2015).

[41] A. Calloni, G. Bussetti, G. Berti, R. Yivlialin, A. Camera, M. Finazzi, L. Duò, and F. Ciccacci, Electronic and magnetic structure of ultra-thin Ni films grown on W(110), J. Magn. Magn. Mater. 420, 356 (2016).

[42] T. Allmers and M. Donath, Growth and morphology of thin $\mathrm{Fe}$ films on flat and vicinal $\mathrm{Au}(111)$ : a comparative study, New J. Phys. 11, 103049 (2009).

[43] G. C. Gazzadi, F. Bruno, R. Capelli, L. Pasquali, and S. Nannarone, Structural transition in Fe ultrathin epitaxial films grown on Ni(111), Phys. Rev. B 65, 205417 (2002).

[44] B. An, L. Zhang, S. Fukuyama, and K. Yokogawa, Growth and structural transition of Fe ultrathin films on $\mathrm{Ni}(111)$ investigated by LEED and STM, Phys. Rev. B 79, 085406 (2009).

[45] F. Bottegoni, A. Calloni, G. Bussetti, A. Camera, C. Zucchetti, M. Finazzi, L. Duò, and F. Ciccacci, Spin polarized surface resonance bands in single layer $\mathrm{Bi}$ on $\mathrm{Ge}(111)$, J. Phys.: Condens. Matter. 28, 195001 (2016).

[46] G. C. Burnett, T. J. Monroe, and F. B. Dunning, Highefficiency retarding-potential Mott polarization analyzer, Rev. Sci. Instrum. 65, 1893 (1994).

[47] I.-G. Baek, H. G. Lee, H.-J. Kim, and E. Vescovo, Spin reorientation transition in $\mathrm{Fe}(110)$ thin films: The role of surface anisotropy, Phys. Rev. B 67, 075401 (2003).

[48] H. L. Meyerheim, D. Sander, R. Popescu, J. Kirschner, O. Robach, and S. Ferrer, Spin Reorientation and Structural Relaxation of Atomic Layers: Pushing the Limits of Accuracy, Phys. Rev. Lett. 93, 156105 (2004).

[49] H. J. Elmers, J. Hauschild, H. Höche, U. Gradmann, H. Bethge, D. Heuer, and U. Köhler, Submonolayer Magnetism of Fe(110) on W(110): Finite Width Scaling of Stripes and Percolation between Islands, Phys. Rev. Lett. 73, 898 (1994).

[50] G. Isella, R. Bertacco, M. Zani, L. Duò, and F. Ciccacci, Evolution of the magnetic and electronic properties of ultrathin Cr(001) films, Solid State Commun. 116, 283 (2000).

[51] M. P. Seah and W. A. Dench, Quantitative electron spectroscopy of surfaces: A standard data base for electron inelastic mean free paths in solids, Surf. Interface Anal. 1, 2 (1979).

[52] G. Rauchbauer, A. Buchsbaum, H. Schiechl, P. Varga, M. Schmid, and A. Biedermann, Ultra-thin Fe films grown on $\mathrm{Cu}$ by pulsed laser deposition: Intermixing and bcc-like structures, Surf. Sci. 602, 1589 (2008).

[53] J. Sánchez-Barriga, J. Fink, V. Boni, I. Di Marco, J. Braun, J. Minár, A. Varykhalov, O. Rader, V. Bellini, F. Manghi, H. Ebert, M. I. Katsnelson, A. I. Lichtenstein, O. Eriksson, W. Eberhardt, and H. A. Dürr, Strength of Correlation Effects in the Electronic Structure of Iron, Phys. Rev. Lett. 103, 267203 (2009).

[54] J. Sánchez-Barriga, J. Braun, J. Minár, I. Di Marco, A. Varykhalov, O. Rader, V. Boni, V. Bellini, F. Manghi, H. Ebert, M. I. Katsnelson, A. I. Lichtenstein, O. Eriksson, W. Eberhardt, 
H. A. Dürr, and J. Fink, Effects of spin-dependent quasiparticle renormalization in $\mathrm{Fe}, \mathrm{Co}$, and $\mathrm{Ni}$ photoemission spectra:An experimental and theoretical study, Phys. Rev. B 85, 205109 (2012).

[55] J. Callaway and C. S. Wang, Energy bands in ferromagnetic iron, Phys. Rev. B 16, 2095 (1977).

[56] J. Redinger, C. L. Fu, A. J. Freeman, U. König, and P. Weinberger, Theoretical spin- and angle-resolved photoemission spectra from Fe(110), Phys. Rev. B 38, 5203 (1988).

[57] A. M. Turner, A. W. Donoho, and J. L. Erskine, Experimental bulk electronic properties of ferromagnetic iron, Phys. Rev. B 29, 2986 (1984).

[58] W. Eberhardt and F. J. Himpsel, Dipole selection rules for optical transitions in the fcc and bcc lattices, Phys. Rev. B 21, 5572 (1980).

[59] F. J. Himpsel, Experimental determination of bulk energy band dispersions, Appl. Opt. 19, 3964 (1980).

[60] A. M. Turner and J. L. Erskine, Exchange splitting and criticalpoint binding energies for iron (110), Phys. Rev. B 25, 1983 (1982).
[61] X.-P. Wang, P. Richard, Y.-B. Huang, H. Miao, L. Cevey, N. Xu, Y.-J. Sun, T. Qian, Y.-M. Xu, M. Shi, J.-P. Hu, X Dai, and H. Ding, Orbital characters determined from Fermi surface intensity patterns using angle-resolved photoemission spectroscopy, Phys. Rev. B 85, 214518 (2012).

[62] A. Calloni, G. Berti, A. Brambilla, G. Bussetti, M. Finazzi, L. Duò, F. Ciccacci, and G. Fratesi, Magnetism in thin $\mathrm{Cr}$ films grown on $\mathrm{Fe}(001)-p(1 \times 1) \mathrm{O}$ : a spin-resolved investigation of single and multi-layers, Proc. SPIE 9551, 95511K (2015).

[63] P. Błoński, A. Kiejna, and J. Hafner, Theoretical study of oxygen adsorption at the $\mathrm{Fe}(110)$ and (100) surfaces, Surf. Sci. 590, 88 (2005).

[64] P. Ohresser, G. Ghiringhelli, O. Tjernberg, N. B. Brookes, and M. Finazzi, Magnetism of nanostructures studied by x-ray magnetic circular dichroism: $\mathrm{Fe}$ on $\mathrm{Cu}(111)$, Phys. Rev. B 62, 5803 (2000).

[65] T. Miller and T.-C. Chiang, Lineshape effects in photoemission from the valence states of metals, J. Phys.: Condens. Matter. 13, 11115 (2001). 